\title{
ON A CUBIC CONGRUENCE IN THREE VARIABLES. II ${ }^{1}$
}

\author{
L. J. MORDELL
}

Let $p$ be a prime and let $f(x, y, z)$ be a cubic polynomial whose coefficients are integers not all $\equiv 0(\bmod p)$, and so are elements of the Galois field $G(p)$. We have the

Conjecture. Suppose that $f(x, y, z)$ cannot be expressed as a cubic polynomial in two independent variables, and that $f(x, y, z)$ is irreducible in any algebraic extension of $G(p)$. Then the number $N$ of solutions of the congruence

$$
f(x, y, z) \equiv 0(\bmod p)
$$

for large p satisfies

$$
N=p^{2}+O(p)
$$

where the constant implied in $O$ is independent of the coefficients of $f(x, y, z)$ and of $p$.

A well-known case when (2) holds is ${ }^{2}$

$$
a x^{3}+b y^{3}+c z^{3}+d \equiv 0,
$$

$a b c d \not \equiv 0$.

Another nontrivial instance is given by [1]

$$
z^{2} \equiv f(x, y)+k
$$

where

$$
f(x, y)=a x^{3}+b x^{2} y+c x y^{2}+d y^{3}
$$

is not a multiple of a perfect cube.

It is not without interest to find other instances for which (2) holds. When I communicated (4) to Professor Davenport, he wrote (October 27, 1961) that (2) also holds for

$$
f(x, y, z) \equiv k,
$$

where $f(x, y, z)$ is the general ternary cubic form.

I prove now the

THEOREM. The result (2) holds for the congruence

$$
z^{2} \equiv f(x, y)+l x+m y,
$$
1962.

Presented to the Society, November 17, 1962; received by the editors April 20,

1 This work was supported in part by the National Science Foundation, Washington, D. C.

${ }^{2}$ We omit $\bmod p$, hereafter in congruences. 
where

$$
f(x, y)=a x^{3}+b x^{2} y+c x y^{2}+d y^{3} \not \equiv g(l x+m y)^{3} .
$$

Proofs of such results are of two kinds. One is at a completely elementary level, but the other makes use of Weil's theorem on the number of solutions of a polynomial congruence $\bmod p$. My proof of (4) was elementary. Professor Davenport in his letter gave a nonelementary proof of (4) and (4a). I give two proofs of the theorem. They both use Weil's results, but the second one, though shorter than the first, requires perhaps more detailed knowledge than the first.

A linear transformation shows that we can replace (5) by

$$
z^{2} \equiv f(x, y)+k x
$$

We note that when $p \equiv 3(\bmod 4), N=p^{2}$.

For clearly

$$
N=\sum_{x, y}\left(1+\left(\frac{f(x, y)+k x}{p}\right)\right),
$$

where the inner bracket denotes the quadratic character $(\bmod p)$. This changes sign when $x, y$ are replaced by $-x,-y$, and so the result follows.

Suppose, hereafter, that $p \equiv 1(\bmod 4)$. We first dispose of the trivial case when $f(x, y)=j x(g x+h y)^{2}$. On replacing $g x+h y$ by $y$, (6) becomes $z^{2} \equiv j x y^{2}+k x$. This congruence has obviously $p^{2}+O(p)$ solutions.

Denote by $N(k)$ the number of solutions of (6). Then $N(k)=N\left(k t^{4}\right)$ where $t \not \equiv 0$ is any integer. For on putting $t^{2} x \equiv X, t^{2} y \equiv Y, t^{3} z \equiv Z$, in $(6)$,

$$
Z^{2} \equiv f(X, Y)+k t^{4} X
$$

Hence $N(k)$ depends only on the biquadratic character of $k(\bmod p)$, and so as $k$ takes all values, $0 \leqq k<p, N(k)$ assumes five values, one $N_{0}$, corresponding to $k=0$, and four others, say $N_{1}, N_{2}, N_{3}, N_{4}$. In (6), the number of solutions with $x \equiv 0$ is $p$, and so we enumerate hereafter only solutions with $x \neq \equiv 0$. Consider now (6) as a congruence in four variables $x \neq 0, y, z, k$. Then we have

$$
N_{0}+\frac{p-1}{4}\left(N_{1}+N_{2}+N_{3}+N_{4}\right)=p^{2}(p-1) .
$$

The left-hand side is the number of solutions corresponding to $k=0,1, \cdots, p-1$. The right-hand side is the number when we take values of $x \neq 0, y, z$, since these define $k$ uniquely. Next 


$$
N_{0}^{2}+\frac{p-1}{4}\left(N_{1}^{2}+N_{2}^{2}+N_{3}^{2}+N_{4}^{2}\right)=p^{5}+E,
$$

where $p^{5}+E$ is the number of solutions of

$$
\frac{z^{2}-f(x, y)}{x} \equiv \frac{z_{1}^{2}-f\left(x_{1}, y_{1}\right)}{x_{1}}
$$

in $x, y, z, x_{1}, y_{1}, z_{1}$ where $x x_{1} \not \equiv 0$.

We show that each side of (8) represents the number of solutions of

$$
z^{2} \equiv f(x, y)+k x, \quad z_{1}^{2} \equiv f\left(x_{1}, y_{1}\right)+k x_{1} .
$$

The left-hand side gives the number for $k=0,1,2, \cdots, p-1$, and the right-hand side the number obtained by equating the two values of $k$. From (7) and (8), since

$$
1+\frac{p-1}{4}(1+1+1+1)=p
$$

we have

$$
\left(N_{0}-p^{2}\right)^{2}+\frac{p-1}{4}\left\{\left(N_{1}-p^{2}\right)^{2}+\cdots+\left(N_{4}-p^{2}\right)^{2}\right\}=E+2 p^{4} .
$$

We shall prove that $E+2 p^{4}=O\left(p^{3}\right)$, and then

$$
N_{1}-p^{2}=O(p)
$$

etc., the required results.

We write (9) as

$$
\frac{z^{2}}{x}-\frac{z_{1}^{2}}{x_{1}} \equiv \frac{f(x, y)}{x}-\frac{f\left(x_{1}, y_{1}\right)}{x_{1}} .
$$

The number of solutions of

$$
A z^{2}+B z_{1}^{2} \equiv C, \quad A B C \not \equiv 0,
$$

is given by

$$
p-\left(-\frac{A B}{P}\right)
$$

If, however, $C \equiv 0$ and $A B \not \equiv 0$, the number is given by

$$
p+(p-1)\left(\frac{-A B}{p}\right) \text {. }
$$


The bracket denotes the quadratic character.

Hence the number $E+p^{5}$ of solutions of (10) is $S_{1}+S_{2}$, where

$$
S_{1}=\sum_{x, x_{1}, y, y_{1}}\left(p-\left(\frac{x x_{1}}{p}\right)\right)=p^{3}(p-1)^{2}
$$

and

$$
S_{2}=p \sum\left(\frac{x x_{1}}{p}\right)
$$

extended over the solutions of

$$
\frac{f(x, y)}{x}-\frac{f\left(x_{1}, y_{1}\right)}{x_{1}} \equiv 0 .
$$

On noting (11)., it suffices to prove that $S_{2}=O\left(p^{3}\right)$.

On putting $y \equiv v x, y_{1} \equiv v_{1} x_{1}$,

$$
S_{2}=p \sum\left(\frac{x x_{1}}{p}\right)
$$

taken over $x^{2} f(1, v) \equiv x_{1}^{2} f\left(1, v_{1}\right)$.

Now put $x_{1} \equiv t x$. Then,

$$
S_{2}=p(p-1) \sum\left(\frac{t}{p}\right)
$$

taken over the solutions of

$$
f(1, v) \equiv t^{2} f\left(1, v_{1}\right) .
$$

In (12), we consider separately the parts arising according as $t$ is a quadratic residue or nonquadratic residue. We put $t=l u^{2}$ where $l=1$ when $t$ is a quadratic residue, and $l=n$ any fixed nonquadratic residue when $t$ is a nonquadratic residue. We have

$$
S_{2}=p(p-1)\left(N_{1}^{\prime}-N_{2}^{\prime}\right)
$$

where $N_{1}^{\prime}, N_{2}^{\prime}$ are the number of solutions in $u, v, v_{1}$ of

$$
f(1, v) \equiv l^{2} u^{4} f\left(1, v_{1}\right)
$$

for $l=1, n$ respectively. We shall prove that

$$
N_{1}^{\prime}=p^{2}+O(p), \quad N_{2}^{\prime}=p^{2}+O(p),
$$

and so $S_{2}=O\left(p^{3}\right)$.

The values of $v_{1}$ for which $f\left(1, v_{1}\right) \equiv 0$ give at most $O(p)$ solutions, 
so we need not consider these $v_{1}$ any further. The number of solutions in $u$ of $u^{4} \equiv s(\bmod p)$ can be written as

$$
1+\chi(s)+\chi^{2}(s)+\chi^{3}(s)
$$

where $\chi$ is an obvious biquadratic character $(\bmod p)$. Hence the number of solutions of (15) is given by

$$
\sum_{v, v_{1}}\left(1+\chi(s)+\chi^{2}(s)+\chi^{3}(s)\right), \quad s=f(1, v) / l^{2} f\left(1, v_{1}\right) .
$$

The first term contributes $p^{2}+O(p)$ to $N_{1}^{\prime}$ and $N_{2}^{\prime}$. The second term contributes a sum

$$
\bar{\chi}\left(l^{2}\right) \sum_{v} \chi(f(1, v)) \sum_{v_{1}} \bar{\chi}\left(f\left(1, v_{1}\right)\right)
$$

where $\bar{\chi}$ is the character conjugate to $\chi$.

By Weil's theorem, the congruence $w^{4} \equiv f(1, v)$ has $p+O(\sqrt{ } p)$ solutions since we have excluded the case when $f(1, v)=j(g+h v)^{2}$. It easily follows, as is already known, and follows from an application of Weil's theorem to a result of Davenport [2], that

$$
\sum_{v} \chi(f(1, v))=O(\sqrt{ } p)
$$

for any nonprincipal biquadratic character. Hence the number of solutions of $(15)$ is equal to $p^{2}+O(p)$. This finishes the proof.

We now give another proof of the theorem. We have seen that

$$
\begin{aligned}
N & =\sum_{x, y}\left(1+\left(\frac{f(x, y)+k x}{p}\right)\right) \\
& =p^{2}-p+S,
\end{aligned}
$$

where on putting $y=v x$,

$$
S=\sum_{x, v}\left(\frac{x^{3} f(1, v)+k x}{p}\right) .
$$

We prove that $S=O(p)$.

We can omit the $O(1)$ values of $v$ for which $f(1, v) \equiv 0$ since the sum in $x$ is then zero. Replace $x$ by $x / f(1, v)$. Then

$$
S=\sum_{x, v}\left(\frac{x^{2}+k f(1, v)}{p}\right)\left(\frac{x}{p}\right) .
$$

Write

$$
S_{A}=\sum_{x}\left(\frac{x^{2}+A}{p}\right)\left(\frac{x}{p}\right) .
$$


Let $r, n$ be a fixed quadratic residue and nonquadratic residue $\bmod p$. We have then $A=t A_{1}^{2}$ where $t=r$ or $n$. On replacing $x$ by $A_{1} x$, and denoting $S_{r}, S_{n}$ by $R, N$ respectively,

$$
\begin{aligned}
S_{A} & =\left(\frac{A_{1}}{p}\right) S_{t}=\frac{1}{2}\left(\frac{A_{1}}{p}\right)\left(\left(1+\left(\frac{t}{p}\right)\right) R+\left(1-\left(\frac{t}{p}\right)\right) N\right), \\
2 S_{A} & =\left(\frac{A_{1}}{p}\right)(R+N)+\left(\frac{A_{1} t}{p}\right)(R-N) .
\end{aligned}
$$

It is known that $|R| \leqq 2 \sqrt{ } p, N \leqq 2 \sqrt{ } p$; in fact, it is easily proved, as is known, that $R^{2}+N^{2}=4 p$.

We show now that when $t A_{1}^{2} \equiv k f(1, v)$, then $\sum_{v}\left(A_{1} / p\right)=O(\sqrt{ } p)$. On changing the notation slightly, it suffices to show that if $g(v)$ is a cubic in $v$ which is not of the form $j(g+h v)^{2}$ and $u^{2} \equiv g(v)$, then $\sum_{0}(u / p)=O(\sqrt{ } p)$.

Replace $u$ by $r u^{2}, n u^{2}$ respectively according as $u$ is a quadratic or nonquadratic residue of $p$. Since the number of solutions of $t u^{4} \equiv g(v)$ is $p+O(\sqrt{ } p)$

$$
\sum_{v}\left(\frac{u}{p}\right)=p+O(\sqrt{ } p)-p-O(\sqrt{ } p)=O(\sqrt{ } p) .
$$

This finishes the proof.

\section{BIBLIOGRAPHY}

1. L. J. Mordell, On a cubic congruence in three variables, Acta Arith. 8(1962-1963), 1-9.

2. H. Davenport, On character sums in finite fields, Acta Math. 71 (1939), 99-121.

University of ARIzona AND

ST. John's COLlege 